\title{
关于广义线性模型拟似然估计弱相合性 的几个问题*
}

\author{
张三国 ${ }^{* *}$ 廖 源
}

(中国科学院研究生院数学科学学院, 北京 100049)

摘要 基于响应变量一维时广义线性模型 $\mathrm{E}(y \mid X)=\mu\left(X^{\prime} \beta\right)$ 的拟似然方程 $\sum_{i=1}^{n} X_{i}\left(y_{i}-\right.$ $\left.\mu\left(X_{i}^{\prime} \beta\right)\right)=0$, 研究了其拟似然估计的弱相合性及其他性质. 在误差 $\left\{e_{i}=Y_{i}-\mu\left(X_{i}^{\prime} \beta_{0}\right), 1 \leqslant\right.$ $i \leqslant n\}$ 不相关及其他条件下, 证明了 $\hat{\beta}_{n}-\beta_{0}=O_{p}\left(\underline{\lambda}_{n}^{-1 / 2}\right)$, 其中 $\hat{\beta}_{n}$ 为上述拟似然方程的一个 解, $\beta_{0}$ 为参数 $\beta$ 的真值, $\underline{\lambda}_{n}$ 为矩阵 $S_{n}=\sum_{i=1}^{n} X_{i} X_{i}^{\prime}$ 的最小特征值. 进一步, 在误差独立且不 含渐近退化子列及其他条件下, 证明了上述收玫速度是确切的. 此外, 平行于 Drygas (1976) 关于线性回归模型的一个经典结果, 证明了对于广义线性模型, 为保证拟似然估计的弱相合 性的必要条件是当 $n \rightarrow \infty$ 时, $S_{n}^{-1} \rightarrow 0$.

关键词 广义线性模型 拟似然估计 弱相合性 收敛速度

$\mathrm{MSC}(2000)$ 主题分类 $62 \mathrm{E} 20,62 \mathrm{~J} 12$

\section{1 引言}

广义线性模型 (generalized linear models, GLMs) 由 Nelder 和 Wedderburn ${ }^{[1]}$ 引入. 作为 一种处理非正态响应变量的回归分析模型, 其定义如下:

$$
\left\{\begin{array}{l}
\mu_{i}=\mathrm{E}\left(y_{i} \mid X_{i}\right), \quad i=1, \ldots, n, \\
g\left(\mu_{i}\right)=X_{i}^{\prime} \beta,
\end{array}\right.
$$

其中 $y_{i}$ 是 $q$ 维响应变量, $X_{i}$ 是 $p \times q$ 阶设计阵. 给定 $X_{i}$ 的条件下, $y_{i}(i=1, \ldots, n)$ 独立且有 如下的指数分布:

$$
f\left(y_{i} \mid \theta_{i}\right)=c\left(y_{i}\right) \exp \left(\theta_{i}^{\prime} y_{i}-b\left(\theta_{i}\right)\right)
$$

这里 $\theta_{i}$ 称为自然参数, $b(\cdot)$ 和 $c(\cdot)$ 为相应于指数分布的确定函数. 由此有 $\theta_{i}=\dot{b}^{-1}\left(h\left(X_{i}^{\prime} \beta\right)\right)$, 其中 $\dot{b}$ 为 $b$ 的一阶导数, $\dot{b}^{-1}$ 和 $h$ 分别为 $\dot{b}$ 和 $g$ 的反函数. (1.1) 式中的一一映射 $g(\cdot)$ 充分光 滑, 一般地, 当 $q=1$ 时, $g$ 称作联系函数 (link function). 使得 $\theta_{i}=X_{i}^{\prime} \beta$ 的 $g(\cdot)$ 被称作自然联 系 (canonical link), 即 $g=\dot{b}^{-1}$. 广义线性模型的提出与发展对统计建模产生了深远的影响.

收稿日期: 2006-07-26; 接受日期: 2007-08-22

中国科学院研究生院院长基金和科研启动基金资助项目

* 谨以此文深切怀念陈希孺教授 (1934-2005)

**E-mail: sgzhang@gucas.ac.cn 
例如 Liang 和 Zeger ${ }^{[2,3]}$ 基于广义线性模型提出的可应用于纵向数据的广义估计方程 (GEE) 方法. 广义线性模型也广泛应用到其他模型, 如随机效应模型 ${ }^{[4]}$ 、状态空间模型 ${ }^{[5]}$ 、生存分 析模型 ${ }^{[6]}$.

广义线性模型参数估计一般用极大似然方法. 给定 $n$ 对响应变量与设计阵 $\left\{\left(y_{i}, X_{i}\right), i=\right.$ $1, \ldots, n\}$, 通过极大化似然函数得到 (1.1) 式中未知参数 $\beta$ 的真值 $\beta_{0}$ 的极大似然估计 (MLE). 一般来说广义线性模型的大样本统计推断就是基于 MLE 的渐近性质. 由 (1.1) 和 (1.2) 式, 略 去无关常数, 可得如下对数似然函数 (log-likelihood function):

$$
l_{n}(\beta)=\sum_{i=1}^{n}\left(y_{i}^{\prime} \theta_{i}-b\left(\theta_{i}\right)\right)
$$

及得分函数 (score function)

$$
L_{n}(\beta)=\sum_{i=1}^{n} X_{i} H\left(X_{i}^{\prime} \beta\right) \Sigma^{-1}\left(X_{i}^{\prime} \beta\right)\left(y_{i}-\mu\left(X_{i}^{\prime} \beta\right)\right),
$$

其中 $H(t)=\partial h(t)^{\prime} / \partial t, \Sigma\left(X_{i}^{\prime} \beta\right)=\operatorname{Cov}_{\beta}\left(y_{i}\right)$, 并用 $\mu$ 代替 $h$ 以强调均值函数正确假定. 特别 地, (1.4) 式在自然联系下变成

$$
L_{n}(\beta)=\sum_{i=1}^{n} X_{i}\left(y_{i}-\mu\left(X_{i}^{\prime} \beta\right)\right) .
$$

一般地, 全局的 MLE 是使得 $l_{n}(\beta)$ 在其定义域上全局最大化, 局部的 MLE 是方程 $L_{n}(\beta)=0$ 的一个根 $\hat{\beta}_{n}$, 使得 $-\partial^{2} l_{n}(\beta) / \partial \beta \partial \beta^{\prime}$ 在 $\hat{\beta}_{n}$ 处正定. 全局和局部的 MLE 可能不同, 但它们在 许多情形下是一致的, 例如自然联系. 经典的广义线性模型极大似然估计的大样本性质可参 见文献 [7].

由 (1.1) 和 (1.2) 式定义的经典广义线性模型, 响应变量独立且服从指数分布. 然而在实 际应用中, 观测到的响应变量之间可能存在正相关性, 这就导致了所谓的 “超散布性” (overdispersion). 一种处理这种现象的方法是引入超散布参数. Wedderburn ${ }^{[8]}$ 假定均值和方差被正 确设定为

$$
\mathrm{E}(y \mid X)=\mu=h\left(X^{\prime} \beta\right), \quad \operatorname{Var}(y \mid X)=\sigma^{2}(\mu)=\phi \nu(\mu),
$$

其中 $\nu(\mu)$ 是方差函数 (通常独立于指数族分布定义), $\phi(>0)$ 是超散布参数. 这样就可得到 “拟似然函数” $Q(\beta, \phi)$, 且 $\partial Q / \partial \beta$ (见文献 [8]) 与得分函数 (1.4) 有类似形式. 有关超散布性的 较好的讨论见文献 [9] 第 6 章. Hinde 和 Démetrio ${ }^{[10]}$, Poortema ${ }^{[11]}$, Liang 和 McCullagh [12] 也有关于超散布性的讨论及例子.

虽然解决超散布性是最初提出拟似然方法的重要动机之一, 但是拟似然方法把广义线性 模型推广到更广阔的应用领域. 拟似然方法的提出已经抛弃了响应变量服从指数分布的假 设, 并分离了均值与方差的结构. 事实上, 不需要完全确定响应变量的分布, 只需正确假定其 一阶矩和二阶矩, 就可在适当条件下得到参数的相合估计, 以及估计的其他大样本性质. Liang 和 Zeger ${ }^{[2]}$ 为拟似然方法引入了一种更普遍的, 并被研究和应用至今的方法. 他们提出, 只要 均值函数假定正确, 就可以预先假定响应变量的 “工作分布” (working distribution). 进而用 “工作方差” (working variance) $\Lambda(\cdot)$ 替换得分函数 (1.4) 中的真实方差 $\Sigma(\cdot)$, 并保留响应变量 独立的假设, 便可得到拟似然方程或广义估计方程 (generalized estimating equation, GEE)

$$
L_{n}(\beta)=\sum_{i=1}^{n} X_{i} H\left(X_{i}^{\prime} \beta\right) \Lambda^{-1}\left(X_{i}^{\prime} \beta\right)\left(y_{i}-\mu\left(X_{i}^{\prime} \beta\right)\right) .
$$


方程 $L_{n}(\beta)=0$ 的根 $\hat{\beta}_{n}$ 称作 $\beta_{0}$ 的拟极大似然估计. 此方法的灵活性表现在若错误地假 设密度形式或联系函数, 还可以选择 “工作分布”, 且并不限于响应变量的维数是一维. 而 Wedderburn ${ }^{[8]}$ 的拟似然方法仅适用于一维响应变量的广义线性模型, 而且选定了均值同时 也选定了 “工作方差”, 因此若在某个场合知道响应变量方差的正确形式或部分信息, 将失去 利用它的机会. 一般来说, 不能相对正确地设定 “工作方差” $\Lambda(\cdot)$ 会导致对参数 $\beta$ 的估计效率 的降低. 关于这方面拟似然估计的大样本性质的一些工作可以参见文献 [13].

拟似然估计大样本性质的研究一直受到国内外许多学者的关注. Chen 等 [14] 讨论了固 定设计和自适应设计下, 拟似然估计的强相合性和收玫速度, 但其一关键步骤的正确性值得 商榷. Chang ${ }^{[15]}$ 通过定义 “最后时” (last-time) 随机变量, 得到了自适应设计下拟似然估计 的强相合性. Chiou 和 Müler ${ }^{[16]}$ 把通常似然函数中的方差用其非参形式的估计代替, 来重新 定义非参形式的拟似然函数, 并指出如此得到的参数的渐近分布与方差正确设定情形下得到 的参数估计的渐近分布相同. 陈夏和陈希孺 ${ }^{[17]}$ 通过在特定阶段 $k$ 停止迭代的方法修改了 Chiou 和 Müler ${ }^{[16]}$ 的非参拟似然的定义, 从而使问题得到简化, 避免了由于取极限 $k \rightarrow \infty$ 带来的某些麻烦, 证明了拟似然估计的渐近性质是不变的, 并给出了严谨的数学证明. 岳丽 和陈希預 ${ }^{[18]}$ 证明了在响应变量有正确表述的假定及其他一些光滑性条件下, 当样本量充分 大时, 拟似然方程的解以概率 1 存在, 同时给出了该解收玫于真值的速度, 并指出在一个重要 特例中, 该速度同于独立同分布随机变量序列部分和的重对数律所确定的速度, 故不能改进. 尹长明和赵林城 ${ }^{[19]}$ 减弱了岳丽和陈希預 ${ }^{[18]}$ 给出的条件, 用不同的方法也得到了拟似然估 计的存在性和强相合性.

本文在假定误差方差有界而不假定误差分布, 分别在误差不相关和独立的条件下研究了 响应变量一维时的广义线性模型拟似然估计的弱相合性.

\section{2 主要结果及讨论}

以下先约定: 用 $c, c_{1}$ 或者 $c_{2}$ 表示一般的不依赖 $n$ 的正常数, 在不同的地方其值可以不 同; 随机变量序列 $\left\{\xi_{n}, i \geqslant 1\right\}$ 称为 “几乎处处” 收玫 (a.s.), 若 $P\left(n \rightarrow \infty\right.$ 时 $\xi_{n}$ 收敛 $)=1$.

重述有关符号如下: 给定 $n$ 对一维响应变量与 $p$ 维固定设计阵 $\left\{\left(y_{i}, X_{i}\right), i=1, \ldots, n\right\}$, 为强调期望正确假定, 用 $\mu(\cdot)$ 代替 $h(\cdot)$ 并明确响应变量 $y_{i}$ 与协变量 $X_{i}$ 的关系 $\mathrm{E}\left(y_{i} \mid X_{i}\right)=$ $\mu\left(X_{i}^{\prime} \beta_{0}\right)$, 其中 $\beta_{0}$ 为参数 $\beta$ 的真值. 拟似然估计 $\hat{\beta}_{n}$ 是拟似然方程

$$
L_{n}(\beta) \equiv \sum_{i=1}^{n} X_{i}\left(y_{i}-\mu\left(X_{i}^{\prime} \beta\right)\right)=0
$$

的根. 此外, 记 $\left\{e_{i}=y_{i}-\mu\left(X_{i}^{\prime} \beta_{0}\right), i=1, \ldots, n\right\}$ 为残差, $\mathrm{E}\left(e_{i}\right)=0 ; \underline{\lambda}_{n}\left(\bar{\lambda}_{n}\right)$ 为 $p \times p$ 维正定对 称阵 $S_{n} \equiv \sum_{i=1}^{n} X_{i} X_{i}^{\prime}$ 的最小 (大) 特征值.

列出所需假设条件如下:

1. $\left\{X_{i}, i \geqslant 1\right\}$ 有界.

2. $S_{n}^{-1} \rightarrow 0$, 当 $n \rightarrow \infty$.

3. 存在 $\epsilon>0$ 和相应的闭区间 $J$ 满足

(1) $\left\{X_{i}^{\prime} \beta: i \geqslant 1,\left\|\beta-\beta_{0}\right\| \leqslant \epsilon\right\} \subset J$;

(2) $\mu$ 在 $J$ 上连续, 并存在一阶导数 $\dot{\mu}$ 和二阶导数 $\ddot{\mu}$, 且 $\dot{\mu}$ 在 $J$ 的内部 $J^{o}$ 上连续并对所 有的 $t \in J^{o}, 0<c_{1} \leqslant \dot{\mu}(t) \leqslant c_{2}<\infty$. 
$4_{1}$. $\left\{e_{i}\right\}$ 不相关, 即 $\mathrm{E}\left(e_{i} e_{j}\right)=0$, 对 $i \neq j ; 0<\underline{\sigma}^{2}=\inf _{i \geqslant 1} \sigma_{i}^{2} \leqslant \sup _{i \geqslant 1} \sigma_{i}^{2}=\bar{\sigma}^{2}<$ $\infty$, 这里 $\sigma_{i}^{2}=\operatorname{Var}\left(e_{i}\right)$ 对所有的 $i \geqslant 1$.

$4_{2}$. $\left\{e_{i}\right\}$ 独立且 $0<\underline{\sigma}^{2} \leqslant \bar{\sigma}^{2}<\infty ;\left\{e_{i}\right\}$ 不含渐近退化子列 (随机变量序列 $\left\{\xi_{i}\right\}$ 称为渐 近退化的, 若存在常数序列 $\left\{a_{i}\right\}$, 使得当 $i \rightarrow \infty$ 时, 依概率 $\left.\xi_{i}-a_{i} \rightarrow 0\right)$.

5. $\bar{\lambda}_{n}=O\left(\underline{\lambda}_{n}^{2}\right)$.

我们的主要结果表述为下面的定理 1-3:

定理 1 假定条件 $1-3$ 和 $4_{1}$ 满足, 则拟似然方程 $(2.1)$ 的解 $\hat{\beta}_{n}$ 依概率接近 1 的存在 且 $\hat{\beta}_{n}-\beta_{0}=O_{p}\left(\underline{\lambda}_{n}^{-1 / 2}\right)$, 其中 $\beta_{0}$ 为回归参数的真值.

Chen 等 ${ }^{[14]}$ 试图在条件 $1-3$ 与条件

(A) $\left\{e_{i}\right\}$ 独立且满足

$$
\sum_{i=1}^{\infty} c_{i}^{2}<\infty \Rightarrow \sum_{i=1}^{\infty} c_{i} e_{i} \text { a.s. 收敛 }
$$

下得到 $\hat{\beta}_{n}$ 的强相合性. Gao 和 $\mathrm{Wu}^{[20]}$ 在 $\left\{e_{i}\right\}$ 独立, $\left(\bar{\lambda}_{n} \log \log \bar{\lambda}_{n}\right)^{1 / 2} / \underline{\lambda}_{n} \rightarrow 0(n \rightarrow \infty)$ 及其 他条件下, 通过重对数律估计 $n$ 充分大时 $\sum_{i=1}^{n} X_{i} e_{i}$ 的阶, 从而得到 $\hat{\beta}_{n}$ 的强相合性. 众所周 知, $\left\{e_{i}\right\}$ 满足条件 $(\mathrm{A})$ 不必满足条件 $4_{1}$, 但许多常见情形下满足条件 $(\mathrm{A})$, 例如 $\left\{e_{i}\right\}$ 独立为 鞅差序列, 也满足条件 $4_{1}$. 在下节定理 1 的证明中, 在 $\left\{e_{i}\right\}$ 不相关条件下, 当 $n$ 充分大时, 我 们通过比较 $S_{n}^{-1 / 2} \sum_{i=1}^{n} X_{i}\left[\mu\left(X_{i}^{\prime} \beta\right)-\mu\left(X_{i}^{\prime} \beta_{0}\right)\right]$ 和 $S_{n}^{-1 / 2} \sum_{i=1}^{n} X_{i} e_{i}$ 的阶, 从而得到 $\hat{\beta}_{n}$ 的收玫 速度为 $O_{p}\left(\underline{\lambda}_{n}^{-1 / 2}\right)$.

定理 2 若条件 1-3 和 42 及 5 满足且 $\ddot{\mu}$ 在 $J^{o}$ 中有界, 则上述收玫速度是确切的, 即 $\hat{\beta}_{n}-\beta_{0} \neq o_{p}\left(\underline{\lambda}_{n}^{-1 / 2}\right)$.

一个有趣的问题是定理 2 中的条件 5 是否可以去掉. 对于经典线性回归模型 $(\mu(t)=t)$, 答案是肯定的. 有理由猜想对一般的 $\mu$ (满足条件 3), 条件 5 也可免除, 但证明看来不易. 然 而, 如果响应变量独立且为指数分布, 并且条件 1 和 2 成立, 我们可以得到自然联系下通常极 大似然估计的渐近正态性 (见文献 [7])

$$
F_{n}^{1 / 2}\left(\hat{\beta}_{n}-\beta_{0}\right) \stackrel{d}{\rightarrow} N\left(\mathbf{0}, \mathbf{I}_{p}\right) \text {, 当 } n \rightarrow \infty,
$$

其中 $F_{n}=\sum_{i=1}^{n} X_{i} \Sigma\left(X_{i}^{\prime} \beta_{0}\right) X_{i}^{\prime}$. 在实际应用中, 一种很常见的情形是, 当 $n$ 充分大, 有 $c_{1} I_{p}<$ $S_{n} / n<c_{2} I_{p}$. 进一步可得 $c_{1} I_{p}<F_{n} / n<c_{2} I_{p}$. 显然, 由 (2.2) 式可得 $\hat{\beta}_{n}-\beta_{0} \neq o_{p}\left(\underline{\lambda}_{n}^{-1 / 2}\right)$, 而 此时条件 5 并不成立.

此外, 条件 $4_{2}$ 中关于残差 $\left\{e_{i}, i \geqslant 1\right\}$ 不包含渐近退化子列的假设是不能省去的, 否则, 定理 2 中的收玫速度不再是确切的. 见下例. 考虑线性回归模型

$$
y_{i}=X_{i}^{\prime} \beta_{0}+e_{i} ; X_{i}=1, i \geqslant 1 .
$$

误差 $\left\{e_{i}\right\}$ 独立. 既然条件 5 对于线性模型是不必要的, 那么令 $X_{i}=1$ 以简化问题是合理的. 首先定义 $e_{i}$ 的分布如下:

$$
P\left(e_{i}=0\right)=1-i^{-2}, \quad P\left(e_{i}=i\right)=P\left(e_{i}=-i\right)=\frac{1}{2 i^{2}},
$$

从而 $\mathrm{E}\left(e_{i}\right)=0, \operatorname{Var}\left(e_{i}\right)=1$. 对于充分小的 $\epsilon>0$, 存在充分大的正整数 $k$, 满足

$$
\prod_{i=k+1}^{\infty}\left(1-i^{-2}\right) \geqslant 1-\epsilon
$$


则当 $n>k$ 时有

$$
P\left(\left|\hat{\beta}_{n}-\beta_{0}\right| \leqslant \sum_{i=1}^{k} \frac{i}{n}\right) \geqslant P\left(e_{i}=0, i \geqslant k+1\right) \geqslant 1-\epsilon,
$$

由此有 $\hat{\beta}_{n}-\beta_{0}=O_{p}\left(n^{-1}\right)=O_{p}\left(\underline{\lambda}_{n}^{-1}\right)$. 在此例中, 误差序列 $\left\{e_{i}, i \geqslant 1\right\}$ 为渐近退化的, 而定 理 2 的其他条件满足, 其收玫速度比 $O_{p}\left(\underline{\lambda}_{n}^{-1 / 2}\right)$ 高.

定理 3 若条件 1,3 和 $4_{1}$ 满足, 则当 $n \rightarrow \infty$ 时, $S_{n}^{-1} \rightarrow 0$ 是 $\hat{\beta}_{n}$ 弱相合的必要条件.

Fahrmeir 和 Kaufmann ${ }^{[7]}$ 给出一个自然联系下保证通常的极大似然估计 $\hat{\beta}_{n}$ 相合的重 要条件

$$
\lambda_{\min }\left(F_{n}(\beta)\right) \rightarrow \infty \text {, 当 } n \rightarrow \infty,
$$

其中 $\lambda_{\min }\left(\lambda_{\max }\right)$ 表示对称方阵的最小 (最大) 特征值, $F_{n}(\beta)$ 表示 Fisher 信息阵的期望 $E\left(F_{n, \mathrm{obs}}(\beta)\right)=E\left(-\partial^{2} l_{n}(\beta) / \partial \beta \partial \beta^{\prime}\right)$. 类似地, 在经典线性回归模型中, 在关于误差的较弱 条件下, 一个保证最小二乘估计弱相合 ${ }^{[21]}$ 和强相合 ${ }^{[22]}$ 的必要条件是

$$
\left(\sum_{i=1}^{n} X_{i} X_{i}^{\prime}\right)^{-1} \rightarrow 0 \text {, 当 } n \rightarrow \infty .
$$

定理 3 断言, 这也是保证拟似然估计弱相合性的必要条件.

条件当 $n \rightarrow \infty$ 时, $S_{n}^{-1} \rightarrow 0$ 的必要性依赖于 $\dot{\mu}$ 在 $J^{o}$ 上有界. 例如, 令 $\mu(t)=\sqrt{t}, t \geqslant 0$; $X_{i}=i^{-2 / 3}, i \geqslant 1$. 参数空间为 $\{\beta: \beta \geqslant 0\}$, 误差 $\left\{e_{i}=y_{i}-\mu\left(X_{i}^{\prime} \beta_{0}\right), i \geqslant 1\right\}$ 独立且满足 $\mathrm{E}\left(e_{i}\right)=0, \operatorname{Var}\left(e_{i}\right)=1$. 由 $(2.1)$ 式得

$$
\hat{\beta}_{n}=\xi_{n}^{2}, \xi_{n}=\sum_{i=1}^{n} X_{i} y_{i} / \sum_{i=1}^{n} X_{i}^{3 / 2}
$$

注意到 $\mathrm{E}\left(\xi_{n}\right)=\sqrt{\beta_{0}}$, 当 $n \rightarrow \infty$ 时, $\operatorname{Var}\left(\xi_{n}\right)=\sum_{i=1}^{n} X_{i}^{2} /\left(\sum_{i=1}^{n} X_{i}^{3 / 2}\right)^{2} \rightarrow 0$, 因此当 $n \rightarrow \infty$ 时, 依概率 $\xi_{n} \rightarrow \sqrt{\beta_{0}}$, 从而得到当 $n \rightarrow \infty$ 时, 依概率 $\hat{\beta}_{n} \rightarrow \beta_{0}$. 此处当 $n \rightarrow \infty$ 时, $S_{n}^{-1}=\left(\sum_{i=1}^{n} i^{-4 / 3}\right)^{-1} \nrightarrow 0$.

\section{3 定理的证明}

本节给出定理 1-3 的详细证明.

引理 1 令 $\mathrm{H}$ 为 $\mathbb{R}^{p}$ 到 $\mathbb{R}^{p}$ 的连续单射且 $H\left(\beta_{0}\right)=a$. 记 $B_{\delta}\left(\beta_{0}\right)=\left\{\beta \in \mathbb{R}^{p},\left\|\beta-\beta_{0}\right\| \leqslant\right.$ $\delta\}$ 和 $S_{\delta}\left(\beta_{0}\right)=\partial B_{\delta}\left(\beta_{0}\right)=\left\{x \in \mathbb{R}^{p},\left\|\beta-\beta_{0}\right\|=\delta\right\}$. 则 $\inf _{\beta \in S_{\delta}\left(\beta_{0}\right)}\|H(\beta)-a\| \geqslant r$ 隐含着: 对任 意的 $b \in B_{r}(a)$, 存在 $\beta \in B_{\delta}\left(\beta_{0}\right)$, 使得 $H(\beta)=b$ (见文献 [23], p. 395, 推论 3.2).

注 1 映射 $f: X \rightarrow Y$ 称为单射, 若 $f$ 把 $X$ 中不同元素映成 $Y$ 中不同元素, 即 $f$ 为单 射当且仅当 $\left[f(x)=f\left(x^{\prime}\right)\right] \Rightarrow\left[x=x^{\prime}\right]$ (对于单射的定义可参见文献 [23], p. 13, 倒数第 9 行). 由 $S_{n}>0$ 和 $0<c_{1} \leqslant \dot{\mu} \leqslant c_{2}$ 易得 $S_{n}^{-1 / 2}\left(L_{n}(\beta)-L_{n}\left(\beta_{0}\right)\right)$ 是单射.

\section{定理 1 的证明 对任意满足}

$$
a_{n} \uparrow \infty, \quad a_{n} \underline{\lambda}_{n}^{-1 / 2} \rightarrow 0
$$

的序列 $\left\{a_{n}, n \geqslant 1\right\}$, 令 $\eta_{n}=a_{n} \underline{\lambda}_{n}^{-1 / 2}$ (这里序列 $\left\{a_{n}, n \geqslant 1\right\}$ 的存在性由条件 2 保证). 记 $B_{n}=B_{\eta_{n}}\left(\beta_{0}\right)$ 以及 $\partial B_{n}$ 表示 $B_{n}$ 的边界. 由多变量函数的中值定理 (mean value theorem) (见 文献 [24], 定理 13.9), 得

$$
\left\|S_{n}^{-1 / 2}\left(L_{n}(\beta)-L_{n}\left(\beta_{0}\right)\right)\right\|^{2}
$$




$$
\begin{aligned}
& =\left(\beta_{0}-\beta\right)^{\prime}\left[\sum_{i=1}^{n} \dot{\mu}\left(X_{i}^{\prime} \beta_{n i}\right) X_{i} X_{i}^{\prime}\right] S_{n}^{-1}\left[\sum_{i=1}^{n} \dot{\mu}\left(X_{i}^{\prime} \beta_{n i}\right) X_{i} X_{i}^{\prime}\right]\left(\beta_{0}-\beta\right) \\
& =\left(\beta_{0}-\beta\right)^{\prime} T_{n} S_{n}^{-1} T_{n}\left(\beta_{0}-\beta\right),
\end{aligned}
$$

其中 $T_{n}=\sum_{i=1}^{n} \dot{\mu}\left(X_{i}^{\prime} \beta_{n i}\right) X_{i} X_{i}^{\prime}, \beta_{n i} \in \overline{\beta_{0}, \beta}, 1 \leqslant i \leqslant n$. 此处和以后我们用 $\overline{a, b}$ 表示 $a$ 和 $b$ 之 间的连线.

由条件 3 的 (2), 有 $c_{1} T_{n}^{-1} \leqslant S_{n}^{-1} \leqslant c_{2} T_{n}^{-1}$, 因此由 (3.2) 式, 对 $\beta \in \partial B_{n}$,

$$
\begin{aligned}
\left\|S_{n}^{-1 / 2}\left(L_{n}(\beta)-L_{n}\left(\beta_{0}\right)\right)\right\|^{2} & \geqslant c_{1}\left(\beta_{0}-\beta\right)^{\prime} T_{n} T_{n}^{-1} T_{n}\left(\beta_{0}-\beta\right) \\
& \geqslant c_{1}^{2}\left(\beta_{0}-\beta\right)^{\prime} S_{n}\left(\beta_{0}-\beta\right) \\
& \geqslant c_{1}^{2} \underline{\lambda}_{n}\left\|\beta_{0}-\beta\right\|^{2}=c_{1}^{2} \underline{\lambda}_{n} \eta_{n}^{2} .
\end{aligned}
$$

从而对 $\beta \in \partial B_{n}$, 当 $n \rightarrow \infty$ 时,

$$
\left\|S_{n}^{-1 / 2}\left(L_{n}(\beta)-L_{n}\left(\beta_{0}\right)\right)\right\| \geqslant c_{1} \underline{\lambda}_{n}^{1 / 2} \eta_{n}=c_{1} a_{n} \rightarrow \infty .
$$

另一方面, 有

$$
\begin{aligned}
\mathrm{E}\left\|S_{n}^{-1 / 2} L_{n}\left(\beta_{0}\right)\right\|^{2} & =\mathrm{E}\left[\left(S_{n}^{-1 / 2} L_{n}\left(\beta_{0}\right)\right)^{\prime}\left(S_{n}^{-1 / 2} L_{n}\left(\beta_{0}\right)\right)\right] \\
& =\operatorname{tr} \mathrm{E}\left[\left(S_{n}^{-1 / 2} L_{n}\left(\beta_{0}\right)\right)\left(S_{n}^{-1 / 2} L_{n}\left(\beta_{0}\right)\right)^{\prime}\right] \\
& =\operatorname{tr}\left[S_{n}^{-1 / 2}\left(\sum_{i=1}^{n} \sigma_{i}^{2} X_{i} X_{i}^{\prime}\right) S_{n}^{-1 / 2}\right] \\
& \leqslant \operatorname{tr}\left[S_{n}^{-1 / 2}\left(c S_{n}\right) S_{n}^{-1 / 2}\right]=c p .
\end{aligned}
$$

这意味着

$$
S_{n}^{-1 / 2} L_{n}\left(\beta_{0}\right)=O_{p}(1) .
$$

由 (3.3) 和 (3.4) 式, 对 $\beta \in \partial B_{n}$, 存在序列 $\left\{\epsilon_{n}, n \geqslant 1\right\}$ 满足 $\epsilon_{n} \downarrow 0$ 且

$$
P\left(\left\{\inf _{\beta \in \partial B_{n}}\left\|S_{n}^{-1 / 2}\left(L_{n}(\beta)-L_{n}\left(\beta_{0}\right)\right)\right\|>\left\|S_{n}^{-1 / 2} L_{n}\left(\beta_{0}\right)\right\|\right\}\right) \geqslant 1-\epsilon_{n} .
$$

再由引理 1 , 以不小于 $1-\epsilon_{n}$ 的概率, (2.1) 式存在一解 $\hat{\beta}_{n} \in B_{n}$. 此外对任意满足 (3.1) 式的 序列 $\left\{a_{n}\right\}, \hat{\beta}_{n}-\beta_{0}=O_{p}\left(a_{n} \underline{\lambda}_{n}^{-1 / 2}\right)$, 因此 $\hat{\beta}_{n}-\beta_{0}=O_{p}\left(\underline{\lambda}_{n}^{-1 / 2}\right)$.

引理 2 假设独立随机变量序列 $\left\{\xi_{i}, i \geqslant 1\right\}$ 不含渐近退化的子列. 设 $c_{n 1}, \ldots, c_{n n}$ 和 $b_{n}$ 为常数, 使得当 $n \rightarrow \infty$ 时, 依概率 $\sum_{i=1}^{n} c_{n i} \xi_{i}-b_{n} \rightarrow 0$, 则当 $n \rightarrow \infty$ 时,

$$
\sum_{i=1}^{n} c_{n i}^{2} \rightarrow 0
$$

(见文献 [25], p. 53, 定理 2.4).

定理 2 的证明 设 $\hat{\beta}_{n}-\beta_{0}=o_{p}\left(\underline{\lambda}_{n}^{-1 / 2}\right)$, 则存在常数序列 $\epsilon_{n}^{\prime} \downarrow 0$, 使得

$$
\left\|\hat{\beta}_{n}-\beta_{0}\right\| \leqslant \epsilon_{n}^{\prime} \underline{\lambda}_{n}^{-1 / 2}
$$

以不小于 $1-\epsilon_{n}^{\prime}$ 的概率成立. 记 $R_{n}=\sum_{i=1}^{n} \dot{\mu}\left(X_{i}^{\prime} \hat{\beta}_{n i}\right) X_{i} X_{i}^{\prime}, \hat{\beta}_{n i} \in \overline{\beta_{0}, \hat{\beta}_{n}}(1 \leqslant i \leqslant n)$ 和 $S_{n}^{*}=$ $\sum_{i=1}^{n} \dot{\mu}\left(X_{i}^{\prime} \beta_{0}\right) X_{i} X_{i}^{\prime}$. 由 (3.5) 式以及 $\ddot{\mu}(\cdot)$ 在 $J^{o}$ 上有界, 存在常数序列 $\epsilon_{n}^{\prime \prime} \downarrow 0$, 使得

$$
-\epsilon_{n}^{\prime \prime} \underline{\underline{\lambda}}_{n}^{-1 / 2} S_{n}^{*} \leqslant R_{n}-S_{n}^{*} \leqslant \epsilon_{n}^{\prime \prime} \underline{\underline{-}}_{n}^{-1 / 2} S_{n}^{*}
$$

等价于

$$
-\epsilon_{n}^{\prime \prime} \underline{\lambda}_{n}^{-1 / 2} R_{n}^{-1} \leqslant S_{n}^{*-1}-R_{n}^{-1} \leqslant \epsilon_{n}^{\prime \prime} \underline{\lambda}_{n}^{-1 / 2} R_{n}^{-1}
$$


以不小于 $1-\epsilon_{n}^{\prime \prime}$ 的概率成立. 再由 $R_{n}^{-1} \leqslant c S_{n}^{*-1}$, 有

$$
-c \epsilon_{n}^{\prime \prime} \underline{\lambda}_{n}^{-1 / 2} S_{n}^{*-1} \leqslant R_{n}^{-1}-S_{n}^{*-1} \leqslant c \epsilon_{n}^{\prime \prime} \underline{\lambda}_{n}^{-1 / 2} S_{n}^{*-1}
$$

以不小于 $1-\epsilon_{n}^{\prime \prime}$ 的概率成立. 又由于 $\lambda_{\min }\left(S_{n}^{*}\right) \geqslant c_{1} \underline{\lambda}_{n}$, 对任意 $p$ 维单位向量 $a_{n}$,

$$
\left\|a_{n}^{\prime}\left(R_{n}^{-1}-S_{n}^{*-1}\right)\right\| \leqslant c c_{1}^{-1} \epsilon_{n}^{\prime \prime} \underline{\lambda}_{n}^{-3 / 2}
$$

以不小于 $1-\epsilon_{n}^{\prime \prime}$ 的概率成立. 由 (3.7) 式, 条件 5 以及 $\lambda_{\max }\left(S_{n}^{*}\right) \leqslant c_{2} \bar{\lambda}_{n}$, 可得

$$
\left\|a_{n}^{\prime}\left(R_{n}^{-1}-S_{n}^{*-1}\right) S_{n}^{* 1 / 2}\right\|=o_{p}\left(\underline{\lambda}_{n}^{-3 / 2} \bar{\lambda}_{n}^{1 / 2}\right)=o_{p}\left(\underline{\lambda}_{n}^{-1 / 2}\right),
$$

即

$$
\underline{\lambda}_{n}^{1 / 2} a_{n}^{\prime}\left(R_{n}^{-1}-S_{n}^{*-1}\right) S_{n}^{* 1 / 2}=o_{p}(1) .
$$

另一方面, 由 $L_{n}\left(\hat{\beta}_{n}\right)=0$ 有

$$
\begin{aligned}
-L_{n}\left(\beta_{0}\right) & =L_{n}\left(\hat{\beta}_{n}\right)-L_{n}\left(\beta_{0}\right)=\sum_{i=1}^{n} X_{i}\left(\mu\left(X_{i}^{\prime} \beta_{0}\right)-\mu\left(X_{i}^{\prime} \hat{\beta}_{n}\right)\right) \\
& =R_{n}\left(\beta_{0}-\hat{\beta}_{n}\right) .
\end{aligned}
$$

这样, 对任意 $p$ 维单位向量 $a_{n}$,

$$
\begin{aligned}
\underline{\lambda}_{n}^{1 / 2} a_{n}^{\prime}\left(\hat{\beta}_{n}-\beta_{0}\right)= & \underline{\lambda}_{n}^{1 / 2} a_{n}^{\prime}\left(R_{n}^{-1}-S_{n}^{*-1}\right) S_{n}^{* 1 / 2} \cdot S_{n}^{*-1 / 2} L_{n}\left(\beta_{0}\right) \\
& +\underline{\lambda}_{n}^{1 / 2} a_{n}^{\prime} S_{n}^{*-1} L_{n}\left(\beta_{0}\right) .
\end{aligned}
$$

由于假定 $\hat{\beta}_{n}-\beta_{0}=o_{p}\left(\underline{\lambda}_{n}^{-1 / 2}\right)$, 故有 $\underline{\lambda}_{n}^{1 / 2} a_{n}^{\prime}\left(\hat{\beta}_{n}-\beta_{0}\right)=o_{p}(1)$. 此外, 由 (3.8) 式, $S_{n}^{*-1 / 2} L_{n}\left(\beta_{0}\right)=$ $O_{p}(1)$ (类似 (3.4) 式) 和 (3.9) 式可得

$$
\underline{\lambda}_{n}^{1 / 2} a_{n}^{\prime} S_{n}^{*-1} L_{n}\left(\beta_{0}\right)=o_{p}(1),
$$

即当 $n \rightarrow \infty$ 时, 依概率有

$$
\sum_{i=1}^{n} \underline{\lambda}_{n}^{1 / 2} a_{n}^{\prime} S_{n}^{*-1} X_{i} e_{i} \rightarrow 0
$$

再由引理 2 , 依概率的对任意单位向量 $a_{n}$ 有

$$
\sum_{i=1}^{n} \underline{\lambda}_{n} a_{n}^{\prime} S_{n}^{*-1} \cdot X_{i} X_{i}^{\prime} \cdot S_{n}^{*-1} a_{n}=\underline{\lambda}_{n} a_{n}^{\prime} S_{n}^{*-1} S_{n} S_{n}^{*-1} a_{n} \rightarrow 0 .
$$

特别取 $a_{n}$ 为对应特征值 $\underline{\lambda}_{n}$ 的单位向量, 由于 $S_{n} \geqslant c_{1} S_{n}^{*}$ 和 $S_{n}^{*-1} \geqslant c_{2} S_{n}^{-1}$ 有

$$
\underline{\lambda}_{n} a_{n}^{\prime} S_{n}^{*-1} S_{n} S_{n}^{*-1} a_{n} \geqslant c \underline{\lambda}_{n} a_{n}^{\prime} S_{n}^{-1} a_{n}=c>0 .
$$

与 $(3.10)$ 式矛盾.

引理 3 对响应变量一维的线性回归模型 $y_{i}=X_{i}^{\prime} \gamma_{0}+e_{i}(1 \leqslant i \leqslant n, n \geqslant 1)$, 其中误差 $\left\{e_{i}\right\}$ 满足条件 $4_{1}, \gamma_{0}$ 的最小二乘估计 (least square estimates, LSE) 为 $\hat{\gamma}_{n}=S_{n}^{-1} \sum_{i=1}^{n} X_{i} y_{i}$ (此 处 $\left.S_{n}=\sum_{i=1}^{n} X_{i} X_{i}^{\prime}\right)$. 若单位向量 $a_{1}, a_{2}, \ldots$ 使得 $\operatorname{Var}\left(a_{n}^{\prime} \hat{\gamma}_{n}\right) \nrightarrow 0$, 则依概率有 $a_{n}^{\prime}\left(\hat{\gamma}_{n}-\gamma_{0}\right) \nrightarrow 0$.

证明 若 $a_{1}=a_{2}=\cdots=a$, 引理的证明见文献 [21]. 对于一般情形, 不妨假设 $a_{n} \rightarrow a$, 否则可以选取子列. 由于

$$
\operatorname{Var}\left(\hat{\gamma}_{n}\right)=\mathrm{E}\left[\left(\hat{\gamma}_{n}-\gamma_{0}\right)\left(\hat{\gamma}_{n}-\gamma_{0}\right)^{\prime}\right]=S_{n}^{-1} \sum_{i=1}^{n} \sigma_{i}^{2} X_{i} X_{i}^{\prime} S_{n}^{-1} \leqslant \bar{\sigma}^{2} S_{n}^{-1},
$$

易见 $\left\{\operatorname{Var}\left(\hat{\gamma}_{n}\right)\right\}$ 是有界序列. 这样, 由当 $n \rightarrow \infty$ 时 $a_{n} \rightarrow a$, 有 $\operatorname{Var}\left(a_{n}^{\prime} \hat{\gamma}_{n}\right)-\operatorname{Var}\left(a^{\prime} \hat{\gamma}_{n}\right) \rightarrow 0$. 因 此由 $\operatorname{Var}\left(a_{n}^{\prime} \hat{\gamma}_{n}\right) \nrightarrow 0$, 可得 $\operatorname{Var}\left(a^{\prime} \hat{\gamma}_{n}\right) \nrightarrow 0$. 从而由上面 $a_{1}=a_{2}=\cdots=a$ 的结果, 可得依概率 
的 $a^{\prime}\left(\hat{\gamma}_{n}-\gamma_{0}\right) \nrightarrow 0$. 另一方面, 由 $a_{n} \rightarrow a$ 及 $\hat{\gamma}_{n}-\gamma_{0}=O_{p}(1)$, 有依概率的 $\left(a_{n}-a\right)^{\prime}\left(\hat{\gamma}_{n}-\gamma_{0}\right) \rightarrow 0$. 综上所述可得依概率的 $a_{n}^{\prime}\left(\hat{\gamma}_{n}-\gamma_{0}\right) \nrightarrow 0$.

定理 3 的证明 相反地, 若 $S_{n}^{-1} \nrightarrow 0$, 但依概率有 $\hat{\beta}_{n} \rightarrow \beta_{0}$. 如有必要可以选取子列, 我 们不妨假设 $\hat{\beta}_{n} \rightarrow \beta_{0}$ a.s.

矩阵 $R_{n}$ 和 $S_{n}^{*}$ 的定义与定理 2 中的证明一样. 我们有

$$
R_{n}-S_{n}^{*}=\sum_{i=1}^{n}\left(\dot{\mu}\left(X_{i}^{\prime} \hat{\beta}_{n i}\right)-\dot{\mu}\left(X_{i}^{\prime} \beta_{0}\right)\right) X_{i} X_{i}^{\prime}, \quad \hat{\beta}_{n i} \in \overline{\hat{\beta}_{n}, \beta_{0}}, 1 \leqslant i \leqslant n .
$$

由条件 3 的 (2) 及 $\hat{\beta}_{n} \rightarrow \beta_{0}$ a.s., 对任意小的 $\delta>0$, 当 $n$ 充分大时以概率 1 的有

$$
-\delta \dot{\mu}\left(X_{i}^{\prime} \beta_{0}\right) \leqslant \dot{\mu}\left(X_{i}^{\prime} \hat{\beta}_{n i}\right)-\dot{\mu}\left(X_{i}^{\prime} \beta_{0}\right) \leqslant \delta \dot{\mu}\left(X_{i}^{\prime} \beta_{0}\right), \quad 1 \leqslant i \leqslant n .
$$

这样, 当 $n$ 充分大时以概率 1 的有

$$
-\delta S_{n}^{*} \leqslant R_{n}-S_{n}^{*} \leqslant \delta S_{n}^{*} .
$$

因此, 由 $R_{n}^{-1} \leqslant c S_{n}^{*-1}$, 可得当 $n$ 充分大时以概率 1 的有

$$
-c \delta S_{n}^{*-1} \leqslant R_{n}^{-1}-S_{n}^{*-1} \leqslant c \delta S_{n}^{*-1},
$$

即

$$
-c \delta I_{n} \leqslant S_{n}^{* 1 / 2}\left(R_{n}^{-1}-S_{n}^{*-1}\right) S_{n}^{* 1 / 2} \leqslant c \delta I_{n} .
$$

由于 $S_{n}^{-1} \nrightarrow 0$, 存在 $l>0$ 和单位向量 $a_{n}$, 使得对任意 $n$,

$$
a_{n}^{\prime} S_{n}^{-1} a_{n} \geqslant l^{2} .
$$

另一方面,

$$
\begin{aligned}
a_{n}^{\prime}\left(\hat{\beta}_{n}-\beta_{0}\right)= & a_{n}^{\prime} S_{n}^{*-1 / 2} \cdot S_{n}^{* 1 / 2}\left(R_{n}^{-1}-S_{n}^{*-1}\right) S_{n}^{* 1 / 2} \cdot S_{n}^{*-1 / 2} L_{n}\left(\beta_{0}\right) \\
& +a_{n}^{\prime} S_{n}^{*-1} L_{n}\left(\beta_{0}\right) .
\end{aligned}
$$

我们已有 $S_{n}^{*-1 / 2} L_{n}\left(\beta_{0}\right)=O_{p}(1)$ 和依概率的 $\hat{\beta}_{n} \rightarrow \beta_{0}$, 再加上 (3.11) 式和 $\left\{a_{n}^{\prime} S_{n}^{*-1}\right\}$ 有 界以及 (3.13) 式, 可得

$$
a_{n}^{\prime} S_{n}^{*-1} L_{n}\left(\beta_{0}\right)=o_{p}(1) .
$$

考虑线性回归模型 $y_{i}^{*}=Z_{i}^{\prime} \gamma_{0}+e_{i}^{*}(i \geqslant 1)$, 其中 $Z_{i}=\left(\dot{\mu}\left(X_{i}^{\prime} \beta_{0}\right)\right)^{1 / 2} X_{i}, e_{i}^{*}=e_{i} /\left(\dot{\mu}\left(X_{i}^{\prime} \beta_{0}\right)\right)^{1 / 2}$. 记 $\gamma_{0}$ 的 LSE 为 $\hat{\gamma}_{n}$, 则由 (3.14) 式,

$$
a_{n}^{\prime}\left(\hat{\gamma}_{n}-\gamma_{0}\right)=a_{n}^{\prime}\left(\sum_{i=1}^{n} Z_{i} Z_{i}^{\prime}\right)^{-1}\left(\sum_{i=1}^{n} Z_{i} e_{i}^{*}\right)=a_{n}^{\prime} S_{n}^{*-1} L_{n}\left(\beta_{0}\right)=o_{p}(1) .
$$

由 (3.12) 式,

$$
\operatorname{Var}\left(a_{n}^{\prime}\left(\hat{\gamma}_{n}-\gamma_{0}\right)\right)=a_{n}^{\prime}\left(S_{n}^{*-1} \sum_{i=1}^{n} \sigma_{i}^{2} Z_{i} Z_{i}^{\prime} S_{n}^{*-1}\right) a_{n} \geqslant c a_{n}^{\prime} S_{n}^{-1} a_{n} \nrightarrow 0, \quad n \rightarrow \infty .
$$

再由引理 3 , 当 $n \rightarrow \infty$ 时, 依概率有

$$
a_{n}^{\prime}\left(\hat{\gamma}_{n}-\gamma_{0}\right) \nrightarrow 0
$$

这与 (3.15) 矛盾.

致谢 作者深切怀念陈希孺教授, 在他的建议下研究此问题. 同时非常感谢 Zhiliang Ying 和 Kani Chen 教授的有益意见和建议以及 Jiahua Chen, 白志东和薛宏旗教授的热心帮助. 最 后感谢审稿者提出的有益意见和建议. 


\section{参 考 文 献}

1 Nelder J A, Wedderburn R W M. Generalized linear model. J Roy Statist Soc Ser A, 135(3): 370-384 (1972)

2 Liang K Y, Zeger S L. Longitudinal data analysis using generalized linear models. Biometrika, 73(1): 13-22 (1986)

3 Zeger S L, Liang K Y. Longitudinal data analysis for discrete and continuous outcomes. Biometrics, 42(1): 121-130 (1986)

4 Diggle P J, Liang K Y, Zeger S L. Analysis of Longitudinal Data. London: Chapman \& Hall, 1994

5 Künsch H R. State space and hidden markov models. In: Barndorff-Nielsen O, Cox DR, Klüppelberg C ed. Complex Stochastic Systems. London: Chapman \& Hall, 2000, 109-173

6 Andersen P K, Borgan $\phi$, Gill R D, et al. Statistical Models Based on Counting Processes. New York: Springer-Verlag, 1993

7 Fahrmeir L, Kaufmann H. Consistency and asymptotic normality of the maximum likelihood estimator in generalized linear models. Ann Statist, 13(1): 342-368 (1985)

8 Wedderburn R W M. Quasi-likelihood functions, generalized linear models, and the Gauss-Newton method. Biometrika, 61(3): 439-447 (1974)

9 Collett D. Modeling Binary Data. London: Chapman \& Hall, 1991

10 Hinde J, Démetrio C. Overdispersion: models and estimation. Comput Statist Data Anal, 27: 151-170 (1998)

11 Poortema K L. On modeling overdispersion of counts. Statist Neerlandica, 53(1): 5-20 (1999)

12 Liang K Y, McCullagh P. Case studies in binary dispersion. Biometrics, 49(2): 623-630 (1993)

13 Fahrmeir L. Maximum likelihood estimation in misspecified generalized linear models. Statistics, 21(4): 487-502 (1990)

14 Chen K, Hu I, Ying Z. Strong consistency of maximum quasi-likelihood estimators in generalized linear models with fixed and adaptive designs. Ann Statist, 27(4): 1155-1163 (1999)

15 Chang Y I. Strong consistency of maximum quasi-likelihood estimate in generalized linear models via a last time. Statist Probab Lett, 45: 237-246 (1999)

16 Chiou J M, Müller H G. Non-parametric quasi-likelihood. Ann Statist, 27(1): 36-64 (1999)

17 Chen X, Chen X R. Adaptive quasi-likelihood estimate in generalized linear models. Sci China Ser A-Math, 48(6): 829-846 (2005)

18 Yue L, Chen X R. Rate of strong consistency of quasi maximum likelihood estimate in generalized linear models. Sci China Ser A-Math, 47(6): 882-893 (2004)

19 Yin C M, Zhao L C. Strong consistency of maximum quasi-likelihood estimates in generalized linear models. Sci China Ser A-Math, 48(8): 1009-1014 (2005)

20 高启兵, 吴耀华. 广义线性回归拟似然估计的强相合性. 数学年刊 A 辑, 25(6): 705-710 (2004)

21 Drygas H. Weak and strong consistency of the least squares estimators in regression models. Prob Theory Related Fields, 34(2): 119-127 (1976)

22 Lai T L, Robbins H, Wei C Z. Strong consistency of least squares estimates in multiple regression II. J Multivariate Anal, 9: 343-361 (1979)

23 Dugundji J. Topology. Boston: Allyn \& Bacon, 1966

24 Fitzpatrick P M. Advanced Calculus: A Course in Mathematical Analysis. Boston: PWS Publishing Company, Thomson Learning, 1996

25 陈希孺. 线性模型参数的估计理论. 北京: 科学出版社, 1985 